\title{
Multi-centre evaluation of the speed-oligo Mycobacteria assay for differentiation of Mycobacterium spp. in clinical isolates
}

\author{
Sabine Hofmann-Thiel ${ }^{1}$, Laziz Turaev ${ }^{2}$, Tarig Alnour ${ }^{1,3}$, Lore Drath $^{4}$, Maria Müllerova ${ }^{5}$ and Harald Hoffmann ${ }^{1 *}$
}

\begin{abstract}
Background: A new DNA line probe assay (Speed-oligo Mycobacteria, Vircell) has been launched for rapid differentiation of Mycobacterium spp. from cultures. Compared to other line-probe assays, Speed-oligo Mycobacteria covers a relatively limited spectrum of species but uses a simpler and faster dip-stick technique. The present multi-centre, multi-country study aimed at evaluating the utility and usability of Speed-oligo Mycobacteria in routine mycobacteriology diagnostics. Results from Speed-oligo Myobacteria were compared to those from Genotype CM (HAIN lifescience, Nehren, Germany), another line-probe assay.

Methods: Speed-oligo Mycobacteria assay was performed in three main steps: 1) DNA extraction from cultured material 2) PCR amplification of the target gene and an internal control and 3) hybridization of the PCR products to specific probes by means of a dip-stick.

Results: Two hundred forty-two clinical isolates were recovered from consecutive positive mycobacterial cultures at two German (IML Gauting, Bioscientia Ingelheim), one Czech (KLINLAB Prague), and at a Sudanese (Khartoum) laboratory. All Mycobacterium species covered by the assay were reliably recognized. The rate of false positive results was $1.2 \%$ and concerned only the species $M$. marinum and $M$. peregrinum. The identification rate, i.e. the proportion of isolates which was correctly differentiated to the level of species or complex by the assay, differed significantly among laboratories being 94.9\%, 90.7\%, and 75.0\% at the study sites IML Gauting, KLINLAB Prague and Bioscientia Ingelheim, respectively. This difference was caused by different spectra of NTM species encountered by the laboratory centres in daily routine diagnostics.

Conclusions: Speed-oligo Mycobacteria assay was proved a rapid and easy-to-perform alternative to conventional line-probe assays. The assay showed excellent sensitivity with regard to identification of genus Mycobacterium and species/complexes covered by the test. However, due to its relatively limited spectrum of taxa, a varying proportion of NTM may not be identified by the assay in daily diagnostics demanding further analyses. The only significant shortcoming in terms of specificity was the misidentification of the clinically relevant species M. marinum.
\end{abstract}

\section{Background}

Whereas tuberculosis (TB) still represents one of the major public health problems in the non-industrialized world, particularly in Africa, South/East-Asia and the countries of the former Soviet-Union [1], its prevalence is steadily declining in the fully industrialized world. Here

\footnotetext{
* Correspondence: harald.hoffmann@asklepios.com

${ }^{1} \mathrm{IML}$ red $\mathrm{GmbH} \&$ synlab Gauting, Supranational Reference Laboratory of Tuberculosis, c/o Asklepios Fachkliniken, Robert-Koch-Allee 2, Gauting, Germany

Full list of author information is available at the end of the article
}

however, infections caused by non-tuberculous mycobacteria (NTM) are becoming more and more relevant in both, incidence and morbidity, partly due to the spread of iatrogenic immunosuppression [2,3]. Since management, treatment, and infection control measures differ dramatically between TB and NTM infections, a rapid and accurate differentiation between these entities is mandatory as soon as a mycobacterial culture turns positive. Additionally, rapid identification of the most frequent NTM species is desirable helping the clinical doctor to assess the relevance of the laboratory findings. 
Most molecular genetic tests differentiating mycobacterial species fulfil this postulation including sequencing of target genes like 16S rRNA gene (rDNA) [4,5], hsp65 gene [6] or 16S-23S intergenic transcribed sequences (ITS) [7] and commercial tests based on gene probes. Among the commercial tests, the gene probe assay AccuProbe (GenProbe, San Diego) was the first one to be developed. The limitation of this fast single-probe test is the relatively small spectrum of Mycobacterium spp. [8,9]. With the introduction of DNA line probe assays (LPA), the simultaneous differentiation of several mycobacterial species in one single test has become possible. INNO-LiPA Mycobacteria v2 (Innogenetics, Ghent, Belgium) and the GenoType Mycobacterium CM and AS (HAIN Lifesciences, Nehren, Germany) are currently among the most widely used LPAs. They have been evaluated extensively with different panels of mycobacterial species [8,10,12-15] and proved easy-to-interpret, sensitive, specific, cost-effective, and in excellent concordances with independent reference methods such as sequence analyses of housekeeping genes. On the other hand, they require higher turnaroundas well as hands-on-times than for example the Accuprobe system.

Recently, a new LPA for the differentiation of mycobacterial species was introduced. The Speed - oligo Mycobacteria ${ }^{\circledR}$ assay (Vircell, Santa Fé Granada, Spain) uses PCR of 16S-23S ITS target sequences and subsequent hybridization to immobilized probes by means of a fast and easy dip stick technique. It detects up to 12 different taxa represented by six specific probes on the strip covering a smaller spectrum than other LPAs. A recent study assessed the performance of the Speed-oligo Myobacteria assay from cultures in a single laboratory in Spain [16] proving good concordance $(97.2 \%)$ to reference methods.

The spectrum and frequency of NTM may vary significantly between regions in Europe and the world [17-20] having potential implications on the validity of the assay in daily diagnostics. Therefore, a multi-centre and multicountry approach was initiated evaluating Speed-oligo Mycobacteria for differentiation and identification of Mycobacterium spp. from cultures in different settings of routine mycobacteriology diagnostics.

\section{Methods}

\section{Clinical isolates}

Clinical isolates $(\mathrm{n}=242)$ included in this study were recovered from consecutive positive mycobacterial cultures originating from routine diagnostic services at four laboratory centres after N-acetyl-cystein (NALC)- $\mathrm{NaOH}$ decontamination. At the European centres IML Gauting, Bioscientia Ingelheim (both Germany) and KLINLAB Prague (Czech Republic), 59 (February-June 2010), 60 (June-September 2009), and 75 (February-July 2010) isolates, respectively, were obtained within the indicated time periods. At the laboratory in Khartoum (Sudan), 53 consecutive sputum samples from smear positive patients (September-December 2009) were decontaminated using (NALC)- $\mathrm{NaOH}$ [21], re-suspended in $1.0 \mathrm{ml}$ phosphate buffer ( $\mathrm{pH}$ 6.8) and shipped to the IML Gauting for growth detection using solid and fluid media (MGIT ${ }^{\mathrm{TM}}$, Becton-Dickinson, Heidelberg, Germany). Of these, 48 MGIT cultures turned positive with acid-fast bacilli and were tested at the IML Gauting by the Sudanese coauthor.

\section{Reference methods}

All isolates were differentiated to the level of species by routine differentiation methods. At all centres, Genotype $\mathrm{CM}$ was the firstly used differentiation assay. In case of a Mycobacterium tuberculosis complex (MTBC) specific band pattern, Genotype MTBC was performed. If Genotype CM yielded another species-specific band pattern the resulting NTM species was assumed as correct-provided that the identification result was in agreement with growth characteristics and morphology of the isolate-and no further tests were performed. If only genus Mycobacterium was identified, Genotype CM was followed by Genotype AS at study sites Bioscientia Ingelheim and KLINLAB Prague, and if necessary partial $16 \mathrm{~S}$ rDNA sequencing, or directly subjected to sequencing at study site IML Gauting. Two Nocardia spp. were not differentiated to the level of species. In cases of discrepant results by Genotype and Speed-oligo assays, the isolate was re-assessed by sequencing for definite species identification.

\section{Speed-oligo mycobacteria assay}

For DNA extraction, a loop-full of bacteria from Loewenstein-Jensen (LJ) slant culture or bacterial sediments from $1 \mathrm{ml}$ of liquid media were re-suspended in $300 \mu \mathrm{l}$ Vircell sample solution and incubated at $95^{\circ} \mathrm{C}$ for $60 \mathrm{~min}$. After centrifugation for $5 \mathrm{~min}$ at $12,000 \times g$, the lysates were directly used for PCR or stored frozen at $-20^{\circ} \mathrm{C}$. For PCR amplification, $15 \mu \mathrm{l}$ of reconstituted ready-to-use PCR mix (provided with the kit) was added to PCR tubes in a DNAfree and PCR amplicon-free pre-amplification area. Then, $10 \mu \mathrm{l}$ of DNA sample was added and PCR amplification $\left(1 \mathrm{~min} 92^{\circ} \mathrm{C}, 40\right.$ cycles consisting of $10 \mathrm{~s} 92^{\circ} \mathrm{C}, 30 \mathrm{~s} 55^{\circ} \mathrm{C}$, $30 \mathrm{~s} 72^{\circ} \mathrm{C}, 1 \mathrm{~min} 72^{\circ} \mathrm{C}$ ) was started. The PCR product was denaturated for $1 \mathrm{~min}$ at $95^{\circ} \mathrm{C}$ and immediately cooled on ice for no more than $1 \mathrm{~min}$. Then, $5 \mu \mathrm{l}$ of PCR product was added to $40 \mu \mathrm{l}$ of pre-heated $\left(55^{\circ} \mathrm{C}\right)$ running solution in a $1.5 \mathrm{ml}$ tube (post-amplification area). The Speed-oligo strip was immediately inserted and results could be interpreted after 5 min of incubation at $55^{\circ} \mathrm{C}$. Results were considered valid, when both internal control lines (PCL, product control line; PCRCL, PCR control line) were positive. Speed-oligo Mycobacteria includes six specific probes (TL1 to TL6) allowing for the identification of members of 
the M. tuberculosis complex (MTBC) (TL4) and frequently encountered NTMs, i.e. M. avium/M. intracellulare/ M. scrofulaceum group (TL5), M. chelonae/M. abscessus complex (TL1), M. kansasii (TL3), M. gordonae (TL2), and $M$. fortuitum (TL6), each of them yielding individual patterns (Table 1). According to the manufacturer, members of the MTBC can show weak TL3 signals in addition to the MTBC specific TL4 probe. A genus specific probe (TL7) additionally allowed for the identification of the genus Mycobacterium.

\section{Results}

Two hundred forty-two clinical isolates were included in the study (Table 2). They originated from four laboratory centres, two in Germany, one in the Czech Republic, and one in the Sudan. The reference methods used identified 77 isolates (31.8\%) as members of the MTBC, 162 (66.9\%) as NTM and three (1.2\%) as species of related genera (two Nocardia spp., one Tsukamurella pulmonis).

\section{Sensitivity and false results}

Speed-oligo Mycobacteria yielded valid results for all 242 study isolates. Two hundred seventeen isolates (89.7\%) belonged to species covered by specific Speed-oligo probes. They yielded the expected patterns and showed complete concordance to the reference method (Table 2). Twenty-five isolates belonged to species not covered by specific Speed-oligo probes. Twenty-two of them (88.0\%) yielded correct results, i.e. 19 NTM isolates were identified as Mycobacterium spp. and three isolates from other genera did not react with any TL probe.

Three NTM isolates (1.2\%, one from each European study site) yielded unspecific Speed-oligo signals leading to misidentifications: two $M$. peregrinum isolates showed a weak reaction with TL6 probe and were thus misidentified as $M$. fortuitum; one $M$. marinum isolate reacted with TL3 probe and was thus misidentified as M. kansasii.

Overall, the sensitivity of Speed-oligo Mycobacteriadefined as the proportion of correct identifications of isolates belonging to taxa for which the assay possesses specific probes-was $100 \%$ for the genus specific probe
TL7 (239 of 239 isolates) and 100\% for the species/complex specific probes TL1-6 (217 of 217 isolates). The rate of false results-defined as the proportion of test outcomes leading to misidentifications of species-was $1.2 \%$ for species/complex specific probes (3 of 242 isolates).

\section{Identification rate}

Speed-oligo Mycobacteria identified the species/complex of 217 out of 242 clinical isolates (89.7\%). The spectrum of mycobacterial species and consequently the outcome of Speed-oligo Mycobacteria varied significantly from study site to study site (Table 2). At IML Gauting and KLINLAB Prague, $95.5 \%$ and $85.4 \%$, respectively, of NTM isolates belonged to species which were covered by Speed-oligo probes. At Bioscientia Ingelheim this proportion was only $70 \%$. Among Sudanese samples, it was $100 \%$ since the only NTM species found was $M$. fortuitum which is covered by Speed-oligo Mycobacteria. Consequently, the Speed-oligo identification rate, i.e. the proportion of clinical isolates differentiated to the level of species or complex, was relatively poor at Bioscientia Ingelheim (45 of 60 clinical isolates, $75.0 \%$ ), good at KLINLAB Prague (68 of $75,90.7 \%$ ) and at IML Gauting (56 of 59, 94.9\%) but excellent for the Sudanese isolates (48 of 48, 100.0\%).

\section{Comparison to results of Genotype CM}

At all study sites, Genotype CM was used as primary method for differentiation as soon as a mycobacteriological culture turned positive. Therefore, Genotype CM results were available for all study isolates and allowed us to compare them directly to Speed-oligo results (Table 2). Of 77 MTBC isolates, all yielded identical results with both assays. Further differentiation to the species level (76 MTB, one $M$. bovis BCG) confirmed the identifications by both assays.

Concordance of the assays was $98.7 \%$ with respect to 140 NTM isolates belonging to species covered by Speed-oligo Mycobacteria except the following two discrepancies: Genotype CM did not identify the species of one $M$. intracellulare and one $M$. kansasii isolate and

Table 1 Interpretation of Speed-oligo Mycobacteria results

\begin{tabular}{|c|c|}
\hline Microorganism & Specific line $(s)^{a}$ \\
\hline M. chelonae /M. abscessus complex & TL1 \\
\hline M. gordonae & TL2 \\
\hline M. kansasii & TL3 \\
\hline M. tuberculosis complex (M. tuberculosis, M. africanum, M. bovis, M. microti) & TL4 or TL4 + TL3 \\
\hline M. avium /M. intracellulare /M. scrofulaceum group & TL5 \\
\hline M. fortuitum & TL6 \\
\hline Mycobacterium genus & TL7 \\
\hline
\end{tabular}

a Depending on the mycobacterium present in the sample, signals will be observed in indicated lines. According to the manufacturer, TL4 and TL4 + TL3 are interpreted as $M$. tuberculosis complex. 
Table 2 Multi-centre evaluation of Speed-oligo Mycobacteria and comparison to Genotype CM

\begin{tabular}{|c|c|c|c|c|}
\hline \multirow[t]{2}{*}{ Mycobacterium species $^{a}$} & \multirow[t]{2}{*}{ no. of Strains } & \multicolumn{2}{|r|}{ Speed-oligo results } & \multirow[t]{2}{*}{ Genotype CM results ${ }^{d}$} \\
\hline & & Bands $^{b}$ & Identification $^{c}$ & \\
\hline IML Gauting, Germany & 59 & & & \\
\hline M. tuberculosis ${ }^{\mathrm{e}}$ & 13 & (3), 4,7 & M. tuberculosis complex & M. tuberculosis complex \\
\hline M. avium & 14 & 5,7 & MAIS group & M. avium \\
\hline M. intracellulare & 9 & 5,7 & MAIS group & M. intracellulare \\
\hline M. intracellulare & 1 & 5,7 & MAIS group & Mycobacterium spp. \\
\hline M. kansasii & 7 & 3,7 & M. kansasii & M. kansasii \\
\hline M. kansasii & 1 & 3,7 & M. kansasii & Mycobacterium spp. \\
\hline M. abscessus & 1 & 1,7 & M. chelonae/M. abscessus complex & M. abscessus \\
\hline M. chelonae & 1 & 1,7 & M. chelonae/M. abscessus complex & M. chelonae \\
\hline M. gordonae & 7 & 2,7 & M. gordonae & M. gordonae \\
\hline M. fortuitum & 2 & 6,7 & M. fortuitum & M. fortuitum \\
\hline M. peregrinum & 1 & (6), 7 & M. fortuitum & M. peregrinum \\
\hline M. xenopi & 1 & 7 & Mycobacterium spp. & M. xenopi \\
\hline Tsukamurella pulmonis & 1 & - & no Mycobacterium spp. & Gram+ bacteria, high G+C content \\
\hline Bioscientia Ingelheim, Germany & 60 & & & \\
\hline M. tuberculosis ${ }^{\mathrm{e}}$ & 13 & (3), 4,7 & M. tuberculosis complex & M. tuberculosis complex \\
\hline M. bovis $\mathrm{BCG}^{\mathrm{e}}$ & 1 & (3), 4,7 & M. tuberculosis complex & M. tuberculosis complex \\
\hline M. avium & 4 & 5,7 & MAIS group & M. avium \\
\hline M. intracellulare & 6 & 5,7 & MAIS group & M. intracellulare \\
\hline M. scrofulaceum & 1 & 5,7 & MAIS group & M. scrofulaceum \\
\hline M. kansasii & 1 & 3,7 & M. kansasii & M. kansasii \\
\hline M. chelonae & 3 & 1,7 & M. chelonae/M. abscessus complex & M. chelonae \\
\hline M. gordonae & 14 & 2,7 & M. gordonae & M. gordonae \\
\hline M. fortuitum & 2 & 6,7 & M. fortuitum & M. fortuitum \\
\hline M. celatum & 4 & 7 & Mycobacterium spp. & Mycobacterium spp. \\
\hline M. flavescens & 1 & 7 & Mycobacterium spp. & Mycobacterium spp. \\
\hline M. malmoense & 1 & 7 & Mycobacterium spp & M. malmoense \\
\hline M. montefiorense & 1 & 7 & Mycobacterium spp. & Mycobacterium spp. \\
\hline M. peregrinum & 1 & 6,7 & M. fortuitum & M. peregrinum \\
\hline M. simiae & 3 & 7 & Mycobacterium spp. & Mycobacterium spp. \\
\hline M. xenopi & 2 & 7 & Mycobacterium spp. & M. xenopi \\
\hline Nocardia spp. & 2 & - & no Mycobacterium spp. & n.d. \\
\hline KLINLAB Prague, Czech Rep. & 75 & & & \\
\hline M. tuberculosis $^{\mathrm{e}}$ & 26 & (3), 4,7 & M. tuberculosis complex & M. tuberculosis complex \\
\hline M. bovis $\mathrm{BCG}^{\mathrm{e}}$ & 1 & (3), 4, 7 & M. tuberculosis complex & M. tuberculosis complex \\
\hline M. avium & 13 & 5,7 & MAIS group & M. avium \\
\hline M. intracellulare & 5 & 5,7 & MAIS group & M. intracellulare \\
\hline M. kansasii & 3 & 3,7 & M. kansasii & M. kansasii \\
\hline M. abscessus & 1 & 1,7 & M. chelonae/M. abscessus complex & M. abscessus \\
\hline M. chelonae & 1 & 1,7 & M. chelonae/M. abscessus complex & M. chelonae \\
\hline M. gordonae & 9 & 2,7 & M. gordonae & M. gordonae \\
\hline M. fortuitum & 9 & 6,7 & M. fortuitum & M. fortuitum \\
\hline M. lentiflavum & 2 & 7 & Mycobacterium spp. & Mycobacterium spp. \\
\hline M. marinum & 1 & 3,7 & M. kansasii & M. marinum \\
\hline M. xenopi & 4 & 7 & Mycobacterium spp. & M. xenopi \\
\hline Khartoum, Sudan & 48 & & & \\
\hline
\end{tabular}


Table 2 Multi-centre evaluation of Speed-oligo Mycobacteria and comparison to Genotype CM (Continued)

\begin{tabular}{|c|c|c|c|c|}
\hline M. tuberculosis ${ }^{\mathrm{e}}$ & 16 & $(3), 4,7$ & M. tuberculosis complex & M. tuberculosis complex \\
\hline M. tuberculosis ${ }^{\mathrm{e}}+M$. fortuitum & 7 & $(3), 4,6,7$ & M. tuberculosis complex + M. fortuitum & M. tuberculosis complex + M. fortuitum \\
\hline M. fortuitum & 25 & 6,7 & M. fortuitum & M. fortuitum \\
\hline TOTAL & 242 & & & \\
\hline
\end{tabular}

${ }^{\mathrm{a}}$ Differentiation to species level by use of Genotype CM, Genotype AS, Genotype MTBC or sequencing (16S rDNA or hsp65 gene).

${ }^{\mathrm{b}}$ Numbers in brackets indicate weaker staining.

'MAIS group: M. avium M. intracellulare M. scrofulaceum group.

dIdentification results by Genotype CM are shown. n.d.: not determined.

${ }^{\mathrm{e}}$ All MTBC isolates yielded weak TL3 signals in addition to TL4

differentiated them only to the level of the genus. Noteworthy, all but these two isolates were differentiated to the species level by Genotype CM whereas 58 of 140 isolates were only assigned to groups or complexes of species by Speed-oligo Mycobacteria.

Twenty-two NTM isolates belonged to species not included in Speed-oligo Mycobacteria. Eleven of them were differentiated to the species level by Genotype CM yielding $M$. xenopi $(\mathrm{n}=7), M$. malmoense $(\mathrm{n}=1), M$. marinum $(\mathrm{n}=$ $1)$, and M. peregrinum $(\mathrm{n}=2)$. The remaining NTM isolates belonged to the taxa $M$. celatum, $M$. flavescens, M. montefiorense, M. lentiflavum and M. simiae and were identified neither by Genotype CM nor by Speed-oligo Mycobacteria.

Overall, Genotype CM would have been theoretically able to differentiate 228 out of 242 isolates. However, it differentiated 226 of them correctly resulting in a sensitivity of $98.7 \%$ (Speed-oligo: 100\%). None of the Genotype $\mathrm{CM}$ tests performed lead to misidentification of the species being better than that of Speed-oligo Mycobacteria. Genotype CM yielded identification rates of $97.3 \%$ (73 of 75 isolates), $94.9 \%$ ( 56 of 59 isolates), and $81.7 \%$ ( 49 of 60 isolates), at KLINLAB Prague, IML Gauting, and Bioscientia Ingelheim, respectively.

\section{Evaluation of work processes and time required}

Speed-oligo Mycobacteria has been assessed as easy to perform by the laboratory staff at each of the European centres. In particular, the availability of a ready-to-use PCR mix as well as the dip-stick technique was considered a significant simplification of laboratory work processes. At the IML Gauting the total assay time of Speed-oligo Mycobacteria (when handling five strains) was measured to $3 \mathrm{~h}$ composed of $1 \mathrm{~h} 10 \mathrm{~min}$ for DNA extraction, 10 min for pre-PCR steps, $1 \mathrm{~h} 30 \mathrm{~min}$ for PCR amplification and $10 \mathrm{~min}$ for hybridization by dip-stick technique. Cumulative hands-on-time was estimated to $25 \mathrm{~min}$.

\section{Discussion}

Speed-oligo Myobacteria has been developed for the rapid differentiation of mycobacterial species from cultures. Its major advantage consists in its simple dip stick technique which is directly applied to denaturized amplification products. The dip stick procedure is easy to perform requiring only two pipetting steps thereby decreasing the hands-on time as well as the risk of cross-contaminations. Results can be obtained within about $3 \mathrm{~h}$ with a total hands-on time of $25 \mathrm{~min}$. By comparison, the line probe assays Genotype Mycobacteria CM/AS and INNO-LiPA Mycobacteria require a total time of 5-6 h with hands-on times of about $2 \mathrm{~h}$ $[8,13,22]$.

One of the major limitations of Speed-oligo Mycobacteria is that it differentiates only a relatively small spectrum of Mycobacterium species. This gives rise to lower identification rates compared to similar LPAs like Genotype $\mathrm{CM}$ depending on regional differences regarding the spectrum of encountered NTM species. Among our four study centres, identification rates varied significantly. We observed the best identification rate with the Sudanese samples due to the fact that $M$. fortuitum was the only NTM species found. Generally, M. fortuitum is considered one of the most frequent contaminants recovered from clinical [23] and environmental specimens [24] in Africa. Our Sudanese sputa were transported for approximately 1 week before they reached our laboratory. Probably, some of them were overgrown by $M$. fortuitum during shipment biasing the species distribution in this panel. Considering European laboratories, Speed-oligo Mycobacteria showed a good identification rate of $94.9 \%$ with isolates from IML Gauting being in the range reported for INNO LiPA or Genotype CM assays in the literature $(88-96 \%)[13,22,25]$ and identical to that shown for Genotype CM in the present study. By contrast, identification rates of Speed-oligo Mycobacteria decreased to 90.7\% at KLINLAB Prague and 75\% at Bioscientia Ingelheim while the rates of Genotype CM were significantly better at these sites (97.3\% and $81.7 \%$ ). This was mainly due to the species $M$. xenopi which is not covered by Speed-oligo Mycobacteria. M. xenopi is increasingly encountered throughout the world $[17,18,20]$ reaching relative frequencies of up to $26 \%$ of NTM isolates [20]. In order to meet the demands of an identification system covering the most frequent mycobacterial species, 
incorporation of a $M$. xenopi specific probe should be considered by the manufacturer.

Compared to other line-probe assays, Speed-oligo Mycobacteria differentiates most of the species only to a level of complexes or groups. Current guidelines do not demand the differentiation of the species M. avium and M. intracellulare, although the discrimination to M. scrofulaceum would be desirable [26]. However, the differentiation between $M$. chelonae and M. abscessus is considered essential for decisions on the optimal regimen if treatment is necessary.

Overall, all species included in the Speed-oligo Mycobacteria assay were reliably identified and yielded the expected patterns. Among these isolates, Genotype CM failed to identify one M. intracellulare and one M. kansasii isolate. The latter showed an unspecific Genotype pattern (bands 1, 2, 3, 10, 12, 13) and was attributed to M. kansasii sequevar VI-3 by sequence analysis of the $16 \mathrm{~S}$ rDNA; this sequevar of $M$. kansasii had been previously described to be missed by Genotype CM [14]. Partial 16S rDNA sequencing of the $M$. intracellulare isolate showed identity to $M$. avium complex ATCC 35770 strain sequences corresponding to $M$. intracellulare serovar 18/MAC D [27]. Phylogenetically, M. intracellulare serovar 18 forms a rather unique 16S rDNA pattern [28] and appears to be only rarely isolated from human specimens [29]. To our best knowledge, there are no published data regarding performance of Genotype CM.

Speed-oligo Mycobacteria yielded only three out of 242 isolates $(1.2 \%)$ with false positive reactions. Cross-reaction of M. peregrinum with TL6 has not been described so far but can be explained by the relatedness of $M$. peregrinum and $M$. fortuitum which both belong to the M. fortuitum complex. Cross-reaction of M. marinum with the TL3 probe is in concordance with the observation by QuezelGuerraz et al. [16] and is already mentioned in the user's manual by Vircell Microbiologists. Potential misidentification of $M$. marinum as $M$. kansasii is certainly the major shortcoming of the assay. $M$. marinum regionally reaches relative frequencies of up to $2 \%$ of NTM isolates $[17,19]$. Although infection due to $M$. marinum clinically presents with characteristic cuteneous lesions, misidentification may cause confusion and unnecessary delay of definite diagnosis.

\section{Conclusions}

Our aim was a multi-centre, multi-country evaluation of Speed-oligo Mycobacteria to differentiate Mycobacterium spp. from cultures. Two hundred forty-two clinical isolates obtained at four laboratory centres were included. The Speed-oligo Mycobacteria assay was found to represent a rapid and easy-to-perform alternative to conventional lineprobe assays for laboratories that do not need to differentiate all NTM isolates to the species level. The test is very reliable recognizing species that are covered by specific probes on the dip-stick. The rate of false positive reactions among 242 isolates was as low as $1.2 \%$. However, due to the more limited spectrum of species covered by the assay, a varying proportion of NTM in the diagnostic services may not be identified. In some settings the differentiation down to the species level might be required demanding additional analyses.

\section{Acknowledgements}

We thank Mrs Ludmilla Slutzka and Birgit Venema for excellent technical assistance. We are also grateful to José A. Carrillo and Pablo Mendoza for critical reading of the manuscript. Vircell Microbiologists, Santa Fé Granada, Spain, supported the travel costs of L.T. and has provided the Speed-oligo Mycobacteria assays free of charge.

\section{Author details}

${ }^{1}$ IML red GmbH \& synlab Gauting, Supranational Reference Laboratory of Tuberculosis, c/o Asklepios Fachkliniken, Robert-Koch-Allee 2, Gauting, Germany. ${ }^{2}$ National Reference Laboratory of Tuberculosis, Tashkent, Uzbekistan. ${ }^{3}$ Department of Microbiology, Alzaiem Alazhari University, Khartoum North, Sudan. ${ }^{4}$ Bioscientia Institute of Medical Diagnostics GmbH, Ingelheim, Germany. ${ }^{5}$ KLINLAB, Departments of Microbiology and Molecular Biology TB, synlab Prague, Prague, Czech Republic.

\section{Authors' contributions}

S.H.-T. participated in the design of the study, supervised the laboratory work at the IML Gauting, analysed the data and drafted the manuscript; L.T. and T.A. did most of the experimental work at the IML Gauting; L.D. was responsible for testing of clinical isolates and data analysis at Bioscientia Ingelheim; M.M. was responsible for testing of clinical isolates and data analysis at KLINLAB Prague; H.H. supervised the study, participated in its design and the preparation of the manuscript. All authors read and approved the final manuscript.

\section{Competing interests}

The authors declare that they have no competing interests.

Received: 1 June 2011 Accepted: 19 December 2011

Published: 19 December 2011

\section{References}

1. WHO REPORT 2007: Global Tuberculosis Control. Surveillance, Planning, Financing.[http://www.who.int/tb/publications/global_report/2007/pdf/full. pdf].

2. Tortoli E: Clinical manifestations of nontuberculous mycobacteria infections. Clin Microbiol Infect 2009, 15(10):906-910.

3. Piersimoni C, Scarparo C: Pulmonary infections associated with nontuberculous mycobacteria in immunocompetent patients. Lancet Infect Dis 2008, 8(5):323-334.

4. Kox LF, van Leeuwen J, Knijper S, Jansen HM, Kolk AH: PCR assay based on DNA coding for 165 rRNA for detection and identification of mycobacteria in clinical samples. J Clin Microbiol 1995, 33(12):3225-3233.

5. Patel JB, Leonard DG, Pan X, Musser JM, Berman RE, Nachamkin I: Sequence-based identification of Mycobacterium species using the MicroSeq $50016 \mathrm{~S}$ rDNA bacterial identification system. J Clin Microbiol 2000, 38(1):246-251.

6. Kim HJ, Mun HS, Kim H, Oh EJ, Ha Y, Bai GH, Park YG, Cha CY, Kook YH, Kim BJ: Differentiation of Mycobacterial species by hsp65 duplex PCR followed by duplex-PCR-based restriction analysis and direct sequencing. J Clin Microbiol 2006, 44(11):3855-3862.

7. Roth A, Fischer M, Hamid ME, Michalke S, Ludwig W, Mauch H: Differentiation of phylogenetically related slowly growing mycobacteria based on 16S-23S rRNA gene internal transcribed spacer sequences. $J$ Clin Microbiol 1998, 36(1):139-147.

8. Tortoli E, Nanetti A, Piersimoni C, Cichero P, Farina C, Mucignat G, Scarparo C, Bartolini L, Valentini R, Nista D, et al: Performance assessment 
of new multiplex probe assay for identification of mycobacteria. J Clin Microbiol 2001, 39(3):1079-1084.

9. Scarparo C, Piccoli P, Rigon A, Ruggiero G, Nista D, Piersimoni C: Direct identification of mycobacteria from $\mathrm{MB} / \mathrm{BacT}$ alert $3 \mathrm{D}$ bottles: comparative evaluation of two commercial probe assays. J Clin Microbiol 2001, 39(9):3222-3227.

10. Lebrun L, Weill FX, Lafendi L, Houriez F, Casanova F, Gutierrez MC, Ingrand D, Lagrange P, Vincent V, Herrmann JL: Use of the INNO-LiPAMYCOBACTERIA assay (version 2) for identification of Mycobacterium avium-Mycobacterium intracellulare-Mycobacterium scrofulaceum complex isolates. J Clin Microbiol 2005, 43(6):2567-2574.

11. Russo C, Tortoli E, Menichella D: Evaluation of the new GenoType Mycobacterium assay for identification of mycobacterial species. J Clin Microbiol 2006, 44(2):334-339.

12. Padilla E, Gonzalez V, Manterola JM, Perez A, Quesada MD, Gordillo S, Vilaplana C, Pallares MA, Molinos S, Sanchez MD, et al: Comparative evaluation of the new version of the INNO-LiPA Mycobacteria and genotype Mycobacterium assays for identification of Mycobacterium species from $\mathrm{MB} / \mathrm{BacT}$ liquid cultures artificially inoculated with Mycobacterial strains. J Clin Microbiol 2004, 42(7):3083-3088.

13. Makinen J, Sarkola A, Marjamaki M, Viljanen MK, Soini H: Evaluation of genotype and LiPA MYCOBACTERIA assays for identification of Finnish mycobacterial isolates. J Clin Microbiol 2002, 40(9):3478-3481.

14. Richter E, Rusch-Gerdes S, Hillemann D: Evaluation of the GenoType Mycobacterium Assay for identification of mycobacterial species from cultures. J Clin Microbiol 2006, 44(5):1769-1775.

15. Gitti Z, Neonakis I, Fanti G, Kontos F, Maraki S, Tselentis Y: Use of the GenoType Mycobacterium CM and AS assays to analyze 76 nontuberculous mycobacterial isolates from Greece. J Clin Microbiol 2006, 44(6):2244-2246

16. Quezel-Guerraz NM, Arriaza MM, Avila JA, Sanchez-Yebra Romera WE, Martinez-Lirola MJ: Evaluation of the Speed-oligo(R) Mycobacteria assay for identification of Mycobacterium spp. from fresh liquid and solid cultures of human clinical samples. Diagn Microbiol Infect Dis 2010, 68(2):123-131.

17. Moore JE, Kruijshaar ME, Ormerod LP, Drobniewski F, Abubakar I: Increasing reports of non-tuberculous mycobacteria in England, Wales and Northern Ireland, 1995-2006. BMC Public Health 2010, 10:612.

18. van Ingen J, Bendien SA, de Lange WC, Hoefsloot W, Dekhuijzen PN, Boeree MJ, van Soolingen D: Clinical relevance of non-tuberculous mycobacteria isolated in the Nijmegen-Arnhem region, The Netherlands. Thorax 2009, 64(6):502-506.

19. Cassidy PM, Hedberg K, Saulson A, McNelly E, Winthrop KL: Nontuberculous mycobacterial disease prevalence and risk factors: a changing epidemiology. Clin Infect Dis 2009, 49(12):e124-e129.

20. Marras TK, Chedore P, Ying AM, Jamieson F: Isolation prevalence of pulmonary non-tuberculous mycobacteria in Ontario, 1997 2003. Thorax 2007, 62(8):661-666.

21. Rieder HL, Van Deun A, Kam KM, Kim SJ, Chonde TM, Trebucq A, Urbanczik R: Priorities for Tuberculosis Bacteriology Services in LowIncome Countries. Paris, France; ${ }^{\prime \prime}$ Second 2007.

22. Sarkola A, Makinen J, Marjamaki M, Marttila HJ, Viljanen MK, Soini H: Prospective evaluation of the GenoType assay for routine identification of mycobacteria. Eur J Clin Microbiol Infect Dis 2004, 23(8):642-645.

23. Diguimbaye-Djaibe C, Vincent V, Schelling E, Hilty M, Ngandolo R, Mahamat HH, Pfyffer G, Baggi F, Tanner M, Zinsstag J: Species identification of non-tuberculous mycobacteria from humans and cattle of Chad. Schweiz Arch Tierheilkd 2006, 148(5):251-256.

24. Chilima BZ, Clark IM, Floyd S, Fine PE, Hirsch PR: Distribution of environmental mycobacteria in Karonga District, northern Malawi. App/ Environ Microbiol 2006, 72(4):2343-2350.

25. Makinen J, Marjamaki M, Marttila H, Soini H: Evaluation of a novel strip test, GenoType Mycobacterium CM/AS, for species identification of mycobacterial cultures. Clin Microbiol Infect 2006, 12(5):481-483.

26. Griffith DE, Aksamit T, Brown-Elliott BA, Catanzaro A, Daley C, Gordin F, Holland SM, Horsburgh R, Huitt G, lademarco MF, et al: An official ATS/ IDSA statement: diagnosis, treatment, and prevention of nontuberculous mycobacterial diseases. Am J Respir Crit Care Med 2007, 175(4):367-416.

27. Harmsen D, Rothganger J, Frosch M, Albert J: RIDOM: Ribosomal Differentiation of Medical Micro-organisms Database. Nucleic Acids Res 2002, 30(1):416-417.
28. Boddinghaus B, Wolters J, Heikens W, Bottger EC: Phylogenetic analysis and identification of different serovars of Mycobacterium intracellulare at the molecular level. FEMS Microbiol Lett 1990, 58(2):197-203.

29. Askgaard DS, Giese SB, Thybo S, Lerche A, Bennedsen J: Serovars of Mycobacterium avium complex isolated from patients in Denmark. J Clin Microbiol 1994, 32(11):2880-2882.

\section{Pre-publication history}

The pre-publication history for this paper can be accessed here: http://www.biomedcentral.com/1471-2334/11/353/prepub

doi:10.1186/1471-2334-11-353

Cite this article as: Hofmann-Thiel et al: Multi-centre evaluation of the speed-oligo Mycobacteria assay for differentiation of Mycobacterium spp. in clinical isolates. BMC Infectious Diseases 2011 11:353.

\section{Submit your next manuscript to BioMed Central and take full advantage of:}

- Convenient online submission

- Thorough peer review

- No space constraints or color figure charges

- Immediate publication on acceptance

- Inclusion in PubMed, CAS, Scopus and Google Scholar

- Research which is freely available for redistribution

Submit your manuscript at www.biomedcentral com/submit
Biomed Central 